\title{
Experimental verification for an instrumented spherical indentation technique in determining mechanical properties of metallic materials
}

\author{
Peng Jiang \\ National Astronomical Observatories, Chinese Academy of Sciences, Beijing 100012, China \\ Taihua Zhang ${ }^{\text {a) }}$ and Rong Yang \\ State Key Laboratory of Nonlinear Mechanics (LNM), Institute of Mechanics, Chinese Academy of Sciences, \\ Beijing 100190, China
}

(Received 1 November 2010; accepted 18 January 2011)

\begin{abstract}
A spherical indentation-based method and its numerical verification, which is capable of identifying the plastic properties, have been respectively provided by our previous work [P. Jiang, et al., J. Mater. Res. 24, 1045 (2009)] and [T. Zhang, et al., J. Mater. Res. 24, 3653 (2009)]. To examine its effectiveness for practical application, 10 typical metals were selected to perform experimental verifications. Here, the above method was used in combination with the Oliver-Pharr model to avoid its dependence on the previously known elastic modulus. To obtain reliable results, a reasonable calibration has been performed for the used spherical tip with imperfect shape. Finally, the present verification has shown that the deviations of yield strength and elastic modulus obtained from the indentation tests are at most $40 \%$ but are generally within $25 \%$. And the effect of the difference in constitutive relationships between the ideal model and the actual material on the accuracy of the indentation-based method has also been illustrated.
\end{abstract}

\section{INTRODUCTION}

The instrumented indentation test has become the most successful technique for studying the hardness and modulus of small volumes of materials and thin films. The prospect of extending this technique to estimate plastic properties of materials has come into focus. ${ }^{1,2}$ However, some early studies have shown that the stressstrain curve cannot be uniquely determined from loading and unloading curves produced by a single sharp-tipped conical indenter. ${ }^{3,4}$ Although several methods using multiple sharp-tipped indenters have been developed, ${ }^{5-7}$ operating with multiple indenters is still inconvenient. However, by changing to spherical indentation, it is possible to determine the plastic properties by analyzing the load-depth curve produced by a single indenter. ${ }^{8-13}$ Consequently, a spherical indentation-based method, which is capable of characterizing plastic properties of materials from just the loading curve of one simple spherical indentation test, was developed in our previous work. ${ }^{14,15}$

However, our previous method depended on the previously known elastic modulus, which obviously will

\footnotetext{
a) Address all correspondence to this author.

e-mail: zhangth@lnm.imech.ac.cn

DOI: $10.1557 / \mathrm{jmr} .2011 .51$
}

restrict its application. Fortunately, there are several models available for the extraction of elastic modulus from spherical indenter test data, such as the model proposed by Oliver and Pharr. ${ }^{16}$ But the question still remains whether the Oliver-Pharr model can be efficiently combined with our previous method for determining both elastic and plastic properties. Moreover, it must be stressed that our previous method was derived based on the power law hardening model, which usually differs from the actual material stress-strain curve. As shown by Liu et al., ${ }^{17}$ such a difference will introduce errors into the predicted results, but the extent which these will affect the accuracy of our method for actual metals is still unresolved. Hence, this research presents a rigorous experimental verification by comparing with tensile test data on ten typical metals to validate our indentation-based method.

\section{ANALYSIS METHOD}

\section{A. Yield strain and strain-hardening exponent}

By considering the effect of piling-up on the volume displaced by a hydrostatic core (see Fig. 1), a modified expanding cavity model ${ }^{14}$ was developed to describe the relationship between loading total work $W_{t}$ and plastic properties (yield strain $\varepsilon_{y}$ and strain-hardening exponent $n$ ). 


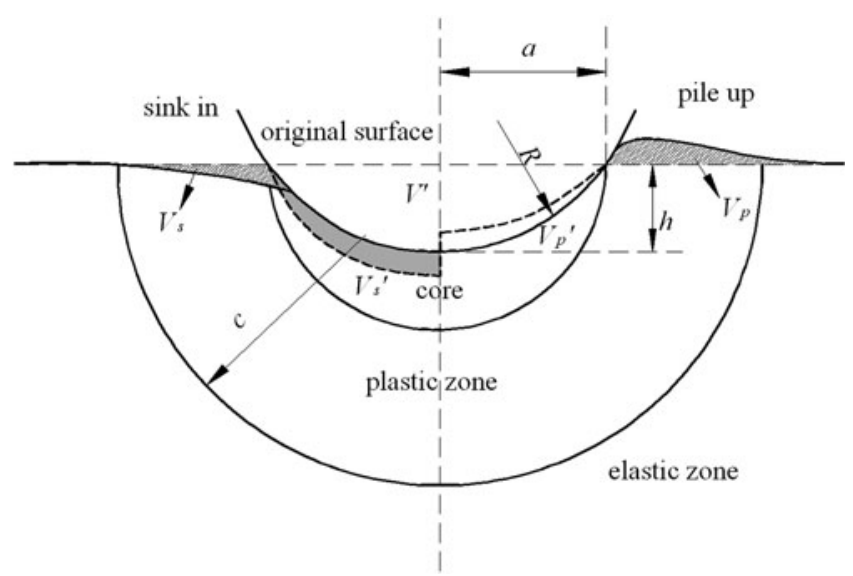

FIG. 1. Schematic of the modified expanding cavity model in which the effect of piling-up or sinking-in is equivalent to some fraction of the hydrostatic core volume displaced by the indenter. ${ }^{14}$

$$
\begin{aligned}
W_{t}= & \frac{2 \pi E \varepsilon_{y}^{2} c^{3}}{3 n(n+1)}\left[\left(\frac{c}{a}\right)^{3 n}-1\right]+\frac{(n-1) \pi E \varepsilon_{y}^{2} a^{3}}{3(n+1)}\left[\left(\frac{c}{a}\right)^{3}-1\right] \\
& +\frac{\pi E \varepsilon_{y}^{2} c^{3}}{3}
\end{aligned}
$$

where $R$ is the radius of the spherical indenter tip, $h$ is the indentation depth, and $a$ is the radius of the hemispherical hydrostatic core, which can be expressed as $a=\sqrt{2 R h-h^{2}}$ (see Fig. 1), $c=\kappa\left[h^{2}(3 R-h) / 3 \varepsilon_{y}\right]^{1 / 3}$ is the radius of elastic-plastic interface, $\varepsilon_{y}$ is the ratio of yield strength to elastic modulus $\left(\sigma_{y} / E\right)$, and $\kappa$ is the correction factor considering the piling up effect, which was given as

$$
\begin{aligned}
\kappa=f\left(\varepsilon_{y}, n\right)= & \left(-0.0077 n^{2}+0.0534 n-0.0304\right) \log ^{2}\left(\varepsilon_{y}\right) \\
& +\left(0.3717 n^{2}-0.1331 n-0.0774\right) \log \left(\varepsilon_{y}\right) \\
& +0.495 n^{2}-0.3016 n+1.0627 .
\end{aligned}
$$

On the other hand, the similarity solution ${ }^{18}$ was investigated numerically in further detail. Then, within a fixed range of $0.6 \leq a / R \leq 0.7$, the relationship between the Meyer index $m$ and plastic properties $\left(\varepsilon_{y}\right.$ and $n$ ) was established as follows ${ }^{14}$

$$
\begin{aligned}
m= & \left(-792.59 n^{2}+1675.9 n-962.01\right) \varepsilon_{y}^{2} \\
& +\left(68.187 n^{2}-112.78 n+57.84\right) \varepsilon_{y}, \\
& -1.4569 n^{2}+2.8637 n+1.7178,
\end{aligned},
$$

where the Meyer index is the gradient of linear regression of $\log F(\operatorname{load} F)$ and $\log a$ of the fixed range of $0.6 \leq a / R$ $\leq 0.7$, which corresponding to the depth range of $0.2 \leq$ $h / R \leq 0.3$.

\section{B. Elastic modulus}

For a given spherical indentation test with specific depth $h=0.3 R, W_{t}$ and $m$ can be obtained from the load-depth curve. Thus, there are three unknown parameters $\left(E, \varepsilon_{y}\right.$, and $\left.n\right)$ remaining in these two equations. Solving for $\varepsilon_{y}$ and $n$ depended on the previously known $E$. Therefore, we now introduce the Oliver-Pharr model ${ }^{16}$ into the above method to solve this problem. The reduced modulus can be expressed as

$$
E_{r}=\frac{S}{2} \sqrt{\frac{\pi}{A\left(h_{c}\right)}},
$$

where $S$ is the slope of the upper portion of the unloading data, which can be obtained from the unloading curve, and $E_{r}$ is the reduced modulus, which can be written as

$$
\frac{1}{E_{r}}=\frac{1-v^{2}}{E}+\frac{1-v_{i}^{2}}{E_{i}},
$$

where $E$ and $v$ are the elastic modulus and Poisson ratio of the specimen, respectively, and $E_{i}$ and $v_{i}$ are the same parameters of the tip. For an ideal spherical tip, the projected contact area $A$ can be derived as

$$
A\left(h_{c}\right)=\pi\left(2 R h_{c}-h_{c}^{2}\right),
$$

where $h_{c}$ is contact depth, which can be determined by Sneddon's solution as

$$
h_{c}=h_{m}-\varepsilon \frac{F_{m}}{S},
$$

with $\varepsilon=0.75$ for a spherical tip. Oliver and Pharr ${ }^{16}$ assumed that the unloading curve obeys

$$
F=a\left(h-h_{f}\right)^{b},
$$

where the constants $h_{f}, A$, and $b$ are all determined by a least squares fitting procedure. From differentiation of the above equation with respect to $h$, the contact stiffness $S$ can be derived as

$$
S=\left.\frac{d F}{d h}\right|_{h=h_{m}}=b a\left(h_{m}-h_{f}\right)^{b-1} .
$$

Once $S$ and $A\left(h_{\mathrm{c}}\right)$ have been obtained from the above procedure, the elastic modulus can be calculated from Eq. (5) by assuming $v=0.3$. Then, by using the Newton iteration method, $\varepsilon_{y}$ and $n$ can be calculated from Eqs. (1) and (3). The above procedure can be summarized as Flow Chart 1 .

\section{EXPERIMENTAL VERIFICATION}

Ten typical metals (Steel IF, Iron DT4, Steel Gr. D, Steel 1045, Al 5083, Al 7075, Al 2024, Copper C11000, Brass C28000, and Ti Grade5) were selected, and tensile 
and indentation tests were performed. These materials cover a wide range of elasticity, from lower elastic material Steel IF to higher one Ti Grade5 (see $\sigma_{y 0}$ in Table I). The effectiveness of this indentation-based method for actual materials can be examined by comparing the materials properties predicted by processing indentation test data and those obtained by tensile tests.

\section{A. Uniaxial tensile tests}

Three dog bone specimens with square cross section $(5 \times 5 \mathrm{~mm})$ were manufactured for each type of material.
The tensile tests were conducted at room temperature using a MTS 810 tester combined with an extensometer (gauge length of $25 \mathrm{~mm}$ ). The tests were performed with a strain rate of $0.01 / \mathrm{s}$. The elastic modulus $\left(E_{0}\right)$, yield strength $\left(\sigma_{y 0}\right)$, and their standard deviations for each material were determined as shown in Table I.

Some materials show obvious Luder's strain behavior, which cannot be fitted well by the ideal power-law expression. In fact, for most common metals, fitting to a different range of the $\sigma-\varepsilon$ curve would lead to different fitted results for the strain hardening exponent. ${ }^{17}$ Hence

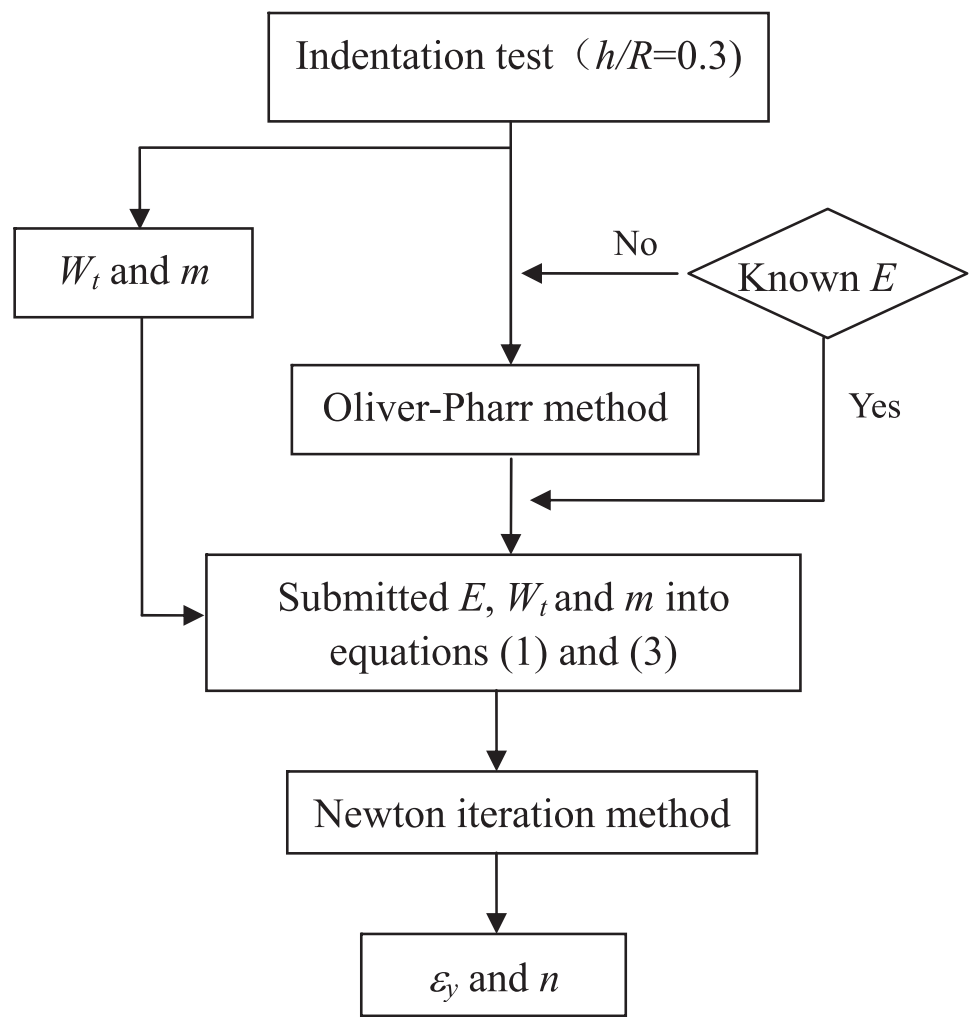

FLOW CHART 1. The procedure of the indentation-based method for obtaining both elastic and plastic properties of a material from a spherical indentation test.

TABLE I. Elastic moduli and yield strengths obtained by tensile tests and predicted from indentation tests for 10 metals.

\begin{tabular}{|c|c|c|c|c|c|c|c|c|c|c|c|}
\hline \multirow[b]{3}{*}{ Material } & \multirow{2}{*}{\multicolumn{2}{|c|}{ Surface texture }} & \multicolumn{4}{|c|}{ Elastic modulus $E(\mathrm{GPa})$} & \multicolumn{4}{|c|}{ Yield strength $\sigma_{y}(\mathrm{MPa})$} & \multirow[b]{3}{*}{$\begin{array}{l}\text { Strain hardening } \\
\quad \text { exponent } n\end{array}$} \\
\hline & & & \multicolumn{2}{|c|}{ Tensile test } & \multicolumn{2}{|c|}{ Indentation test } & \multicolumn{2}{|c|}{ Tensile test } & \multicolumn{2}{|c|}{ Indentation test } & \\
\hline & $R_{a}(\mathrm{~nm})$ & $l_{r}(\mu \mathrm{m})$ & Mean $E_{0}$ & Std. $\Delta E_{0}$ & Mean $E$ & $\begin{array}{c}\text { Deviation } \\
(\%)\end{array}$ & Mean $\sigma_{y 0}$ & Std. $\Delta \sigma_{y 0}$ & Mean $\sigma_{y}$ & $\begin{array}{l}\text { Deviation } \\
(\%)\end{array}$ & \\
\hline Steel IF & 6.9 & 80 & 185.41 & 0.73 & 168.7 & -9.0 & 149.7 & 2.7 & 165.4 & 10.5 & 0.141 \\
\hline Iron DT4 & 93 & 250 & 205.7 & 2.7 & 205.2 & -0.2 & 193.4 & 5.3 & 287.4 & 48.6 & 0.092 \\
\hline Steel Gr.D & 9.0 & 80 & 209.1 & 1.2 & 195.6 & -6.4 & 329.7 & 7.7 & 364.9 & 10.7 & 0.171 \\
\hline Steel 1045 & 9.5 & 80 & 205.1 & 0.85 & 203.6 & -0.7 & 394 & 12 & 403.5 & 2.4 & 0.165 \\
\hline $\mathrm{Al} 5083$ & 47 & 250 & 74.0 & 1.9 & 80.7 & 9.1 & 172.3 & 2.0 & 140.9 & -18.2 & 0.212 \\
\hline Al 7075 & 8.9 & 80 & 70.81 & 0.53 & 67.9 & -4.1 & 410 & 13 & 451.7 & 10.2 & 0.060 \\
\hline Al 2024 & 40 & 250 & 72.91 & 0.57 & 82.3 & 12.8 & 384.7 & 3.6 & 286.7 & -25.5 & 0.187 \\
\hline Copper C11000 & 57 & 250 & 111.6 & 3.2 & 136.3 & 22.1 & 301.5 & 4.2 & 240.2 & -20.3 & 0.092 \\
\hline Brass C28000 & 8.8 & 80 & 100.6 & 1.9 & 65.5 & -34.9 & 173.4 & 1.6 & 229.1 & 32.1 & 0.150 \\
\hline Ti Grade5 & 98 & 250 & 120.47 & 0.99 & 109.4 & -9.2 & 896.4 & 8.1 & 889.3 & -0.8 & 0.104 \\
\hline
\end{tabular}




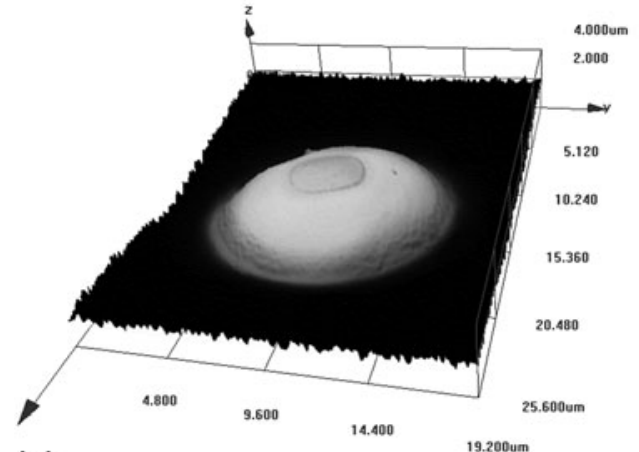

(a)

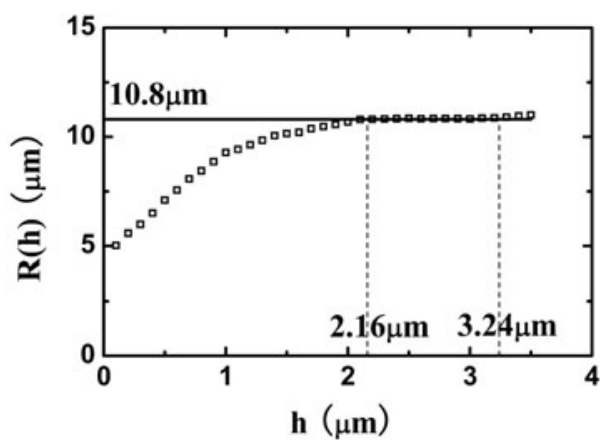

(b)

FIG. 2. (a) The geometrical surface of the tip measured by OLYMPUS OLS3100; (b) the equivalent radius (dots) determined by Eq. (11) and fits for the application range of the indentation-based method (solid line).

we have not given statistics for the strain hardening exponent values. However, the estimated mechanical properties $\left(E, \varepsilon_{y}\right.$, and $\left.n\right)$ of this indentation-based method can be used to predict the $\sigma-\varepsilon$ curve for each metal. Thus, by comparing this predicted $\sigma-\varepsilon$ curve to that obtained from the tensile tests, the accuracy of the estimated strain hardening exponent can be evaluated.

\section{B. Instrumented indentation test}

To assess the validity of this indentation-based method, spherical indentation tests were carried out on the same 10 metals using a Nano Indenter XP (Agilent Technologies, Chandler, AZ) . And a diamond sphero-conical indenter of $10.6 \mu \mathrm{m}$ nominal radius is used in the present experiment. Considering the importance of the indenter radius for the analysis of the test results, the three-dimensional observation of the used tip shape, after cleaning its surface in an ultrasonic bath, was performed using an confocal laser scanning microscope (OLS3100, OLYMPUS, Shinjuku Monolith, Tokyo, Japan), which with vertical resolution of $0.01 \mu \mathrm{m}$ and horizontal resolution $0.12 \mu \mathrm{m}$. The measurement result was shown in Fig. 2(a).

According to the expanding cavity model, the indentation responses are mainly related to the volume of material displaced by the indenter, which is always determined by the volume of indenter itself beneath the material surface. Hence, the volume of a tip, in our opinions, should be an important factor in its calibration. The volume of an ideal sphere beneath the depth $h_{i}$ can be expressed as

$$
V\left(h_{i}\right)=\frac{1}{3} \pi h_{i}^{2}\left[3 R\left(h_{i}\right)-h_{i}\right]
$$

Then, $R\left(h_{i}\right)$ can be solved as

$$
R\left(h_{i}\right)=\frac{V\left(h_{i}\right)}{\pi h_{i}^{2}}+\frac{h_{i}}{3}
$$

Hence, by using the measured data for the volume and height of the tip, $R\left(h_{i}\right)$ can be calculated from Eq. (11) at

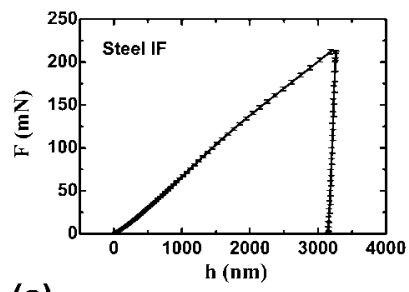

(a)

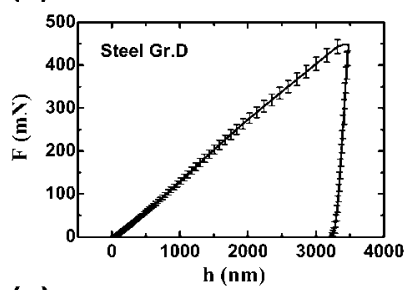

(c)

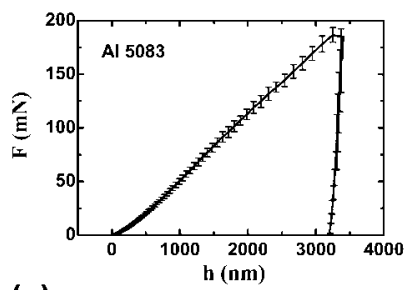

(e)

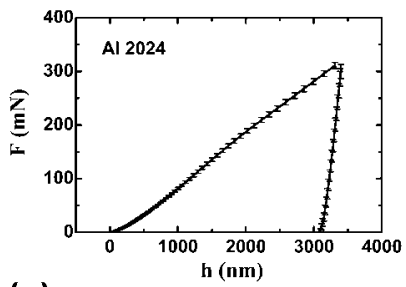

(g)

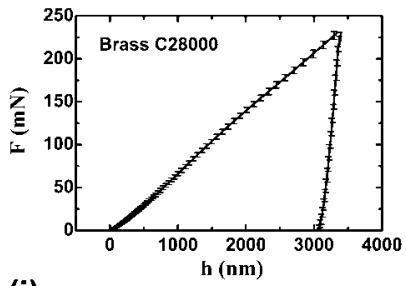

(i)

(b)
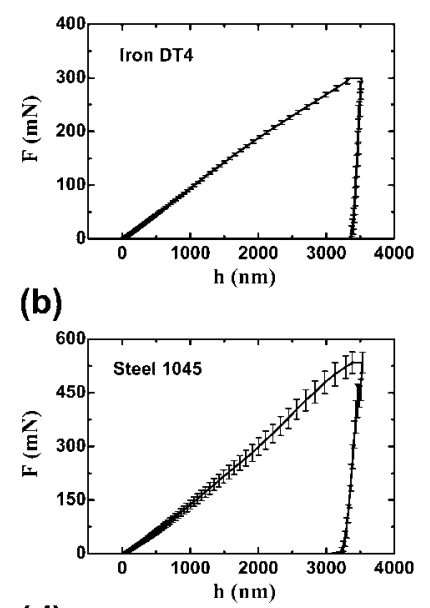

(d)

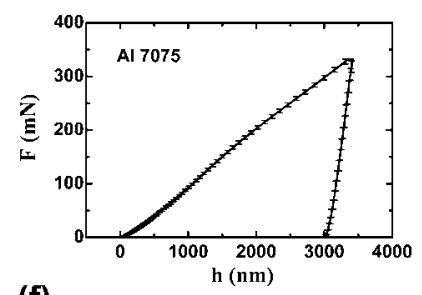

(f)

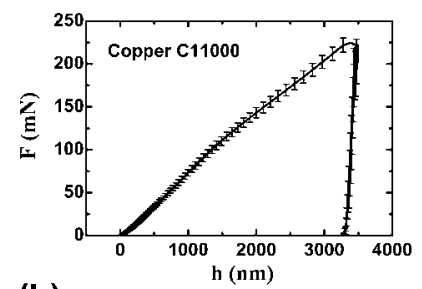

(h)

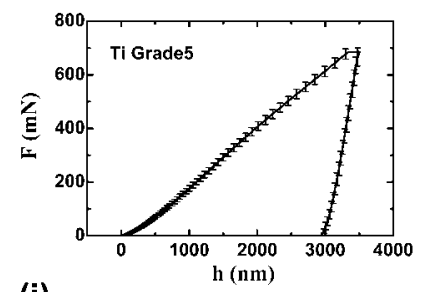

(j)

FIG. 3. Averaged load-depth curves from indentation tests; the error bars span one standard deviation. 

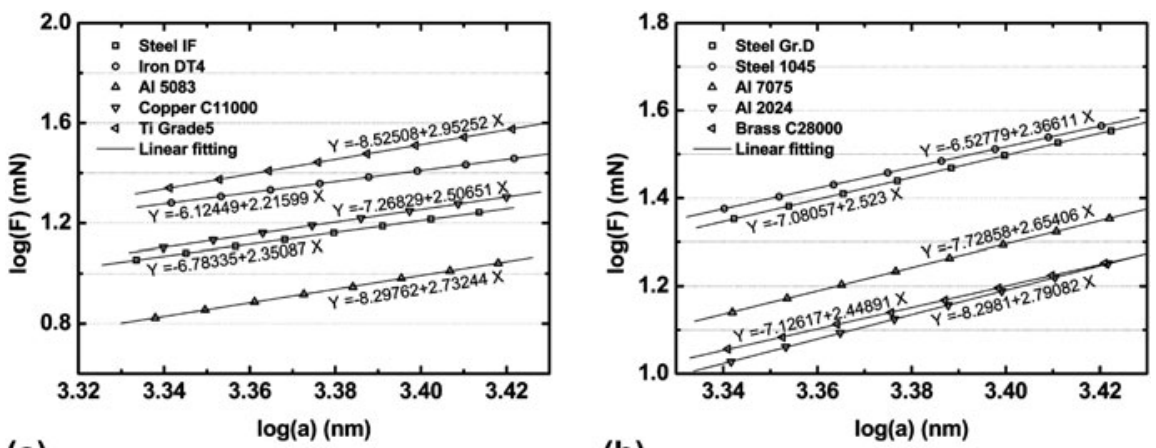

(a)

(b)

FIG. 4. Linear regression of $\log (\mathrm{F})$ versus $\log$ (a) used in the similarity solution.

each depth [see Fig. 2(b)]. It can be readily seen that the equivalent radius increases with indentation depth and approaches a constant value of $10.8 \mu \mathrm{m}$ within a certain range of $h$ (between 2.16 and $3.24 \mu \mathrm{m}$ ), which coincides with the applicable range of the present model. This value of $10.8 \mu \mathrm{m}$ is close to its nominal radius of $10.6 \mu \mathrm{m}$.

Each indentation specimen was taken from the same rod as the tensile specimens for each metal. After mechanically polishing their surfaces, surface roughness of each material was tested with the scratch kit of the Nano Indenter XP with the Berkovich tip. The required inputs are profile load $20 \mu \mathrm{N}$ and profile length $500 \mu \mathrm{m}$. Six tests were performed on each specimen. The surface roughness $R_{a}$ and the roughness sampling length $l_{r}{ }^{19}$ were shown in Table I. The roughness of each specimen is generally less than $100 \mathrm{~nm}$, which is no more than $1 / 30$ of indentation depth $(3.24 \mu \mathrm{m})$, satisfying the requirement in ISO $14577-2002 .^{20}$

All spherical indentation tests were performed with the XP indentation kit of the Nano Indenter XP. The equivalent radius of the spherical tip has been determined as $10.8 \mu \mathrm{m}$ by the previous analysis. For this indentationbased method, the maximum depth of penetration is $h_{\max }=0.3 R$, (i.e., $3.24 \mu \mathrm{m}$ ). Consequently, the maximum depth was fixed to be no less than $3.24 \mu \mathrm{m}$ to ensure that the test data could be used. The required inputs are allowable drift rate $0.03 \mathrm{~nm} / \mathrm{s}$, strain rate target $0.05 / \mathrm{s}$, depth limit $3240 \mathrm{~nm}$, and peak hold time $10 \mathrm{~s}$. Ten tests were performed on each specimen. Figure 3 illustrates the average load-depth data acquired according to the procedure outlined above for each material.

\section{RESULTS AND DISCUSSION}

For a given spherical indentation test, the constants of $h_{f}, A$, and $b$ can be determined based on fitting unloading data well described by Eq. (8) (the correlation coefficients in our procedure are no less than 0.9995). By submitting the value of $h_{f}, a$, and $b$ into Eq. (9), the contact stiffness $S$ can be derived. Then, by using the Oliver-Pharr method, the elastic modulus can be estimated as shown in Table I.

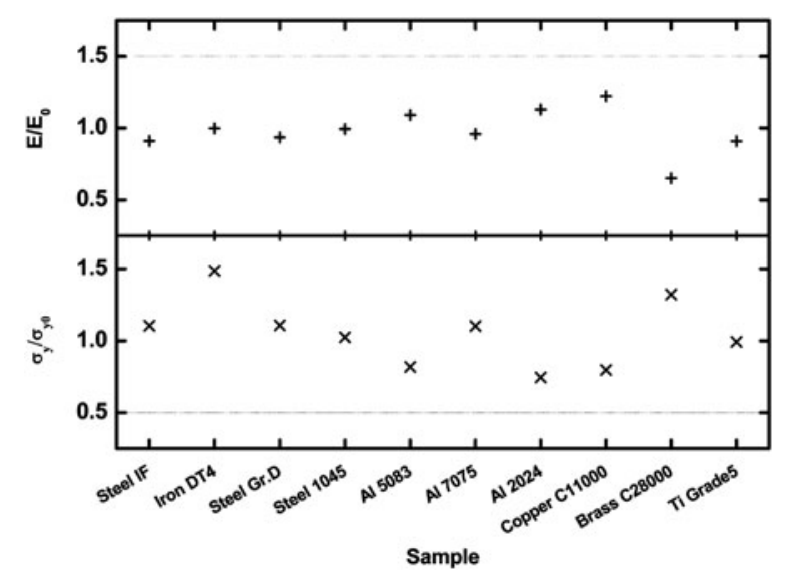

FIG. 5. Comparison of elastic moduli and yield strengths from indentation and tensile tests of 10 metals.

Next, $W_{t}$ and $m$ can be calculated by processing the averaged loading data of load-depth curves, among which $m$ is the gradient of linear regression of $\log F$ and $\log a$ of test data of the fixed range of $0.2 \leq h / R \leq 0.3$, the fitting procedure was illustrated as Fig. 4. By submitting $W_{t}$ and $m$ into Eqs. (1) and (3), $\sigma_{y}$ and $n$ of each material can be solved as shown in Table I. Here the elastic moduli $\left(E_{0}\right)$ and yield strengths $\left(\sigma_{y 0}\right)$ obtained from the tensile tests are conventionally regarded as the true values. In Table I, the deviation percentages of $E$ and $\sigma_{y}$ to their true values are calculated using equations $\left(E-E_{0}\right) / E_{0}$ and $\left(\sigma_{y}-\sigma_{y 0}\right) / \sigma_{y 0}$, respectively.

As shown in Table I and Fig. 5, the deviations of $E$ and $\sigma_{y}$ in most cases are below 25\%, which is sufficiently accurate for most common engineering applications. The predicted results of $E, \varepsilon_{y}$, and $n$, can be used to plot indentation stress-strain curves by the power-law hardening relationship $\sigma=E \varepsilon_{y}^{1-n} \varepsilon^{n}$, as shown in Fig. 6 . Comparing the tensile and indentation stress-strain curves in Fig. 6 shows that the predicted strain hardening exponent approximately describes the hardening characteristics of these metals. Thus, with the $\varepsilon_{y}$ values obtained from the tensile tests and $n$ values predicted by indentation tests, the approximate plastic properties distribution of 


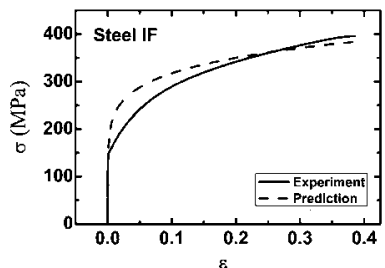

(a)

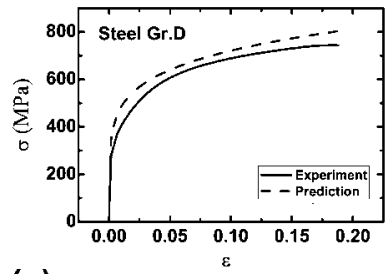

(c)

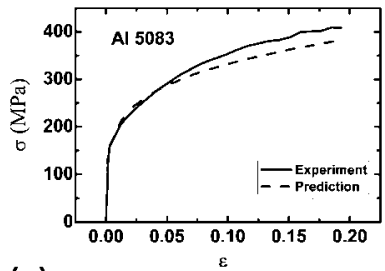

(e)

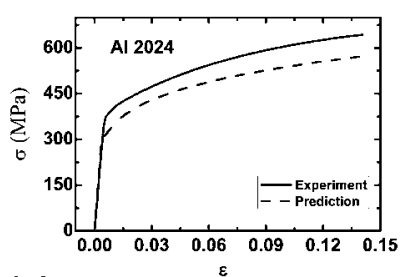

(g)

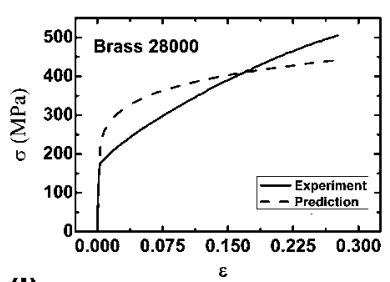

(i)

FIG. 6. Comparison of true stress-strain curves obtained from indentation and tensile tests for 10 metals.

these metals can be summarized in Fig. 7, which shows that these tested materials cover a wide range of mechanical properties.

However, it also should be noted that the maximum deviation for the indentation plastic properties is about $48.6 \%$ for Iron DT4. From its tensile strain-stress curve in Fig. 6(b), Iron DT4 shows obvious Luder's strain, and such a curve cannot be fitted well by the ideal power-law hardening relationship. As illustrated by a recent study, ${ }^{17}$ the difference in constitutive relationship between the ideal model and an actual material does will affect the accuracy of the indentation-based method. Similarly, the deviation of indentation yield strength is also high for

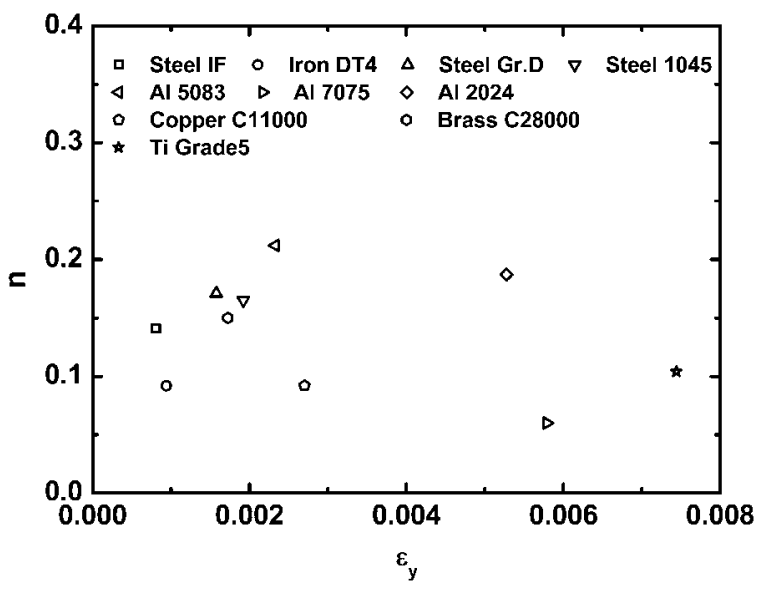

FIG. 7. The distribution of yield strains and strain hardening exponents for 10 metals.

Brass C28000 (32.1\%) because its constitutive relationship is closer to a linear hardening relation than a power law hardening one.

\section{SUMMARY AND CONCLUSIONS}

By combining the Oliver-Pharr model which can determine the elastic properties of materials with our previous method, three elastic and plastic properties can be derived from one simple spherical indentation test. The methodology involves only one indenter, thus the test procedure is greatly simplified and the inconvenience of switching indenters during the test can be avoided. Finally, 10 typical metals were selected for experimental verification, whose results show that this method can provide reasonable estimates of the elastic and plastic properties for most common metals.

However, it should be noted that this indentation-based method must be used with caution for certain metals, such as Iron DT4 and C28000, whose constitutive relationship is not fitted well by a power law hardening model. In addition, the indentation size effect also should be aware if smaller indenter radius is used. These issues will be examined in future studies.

\section{ACKNOWLEDGMENT}

We acknowledge the support of the National Natural Science Foundation of China (Grant Nos. 11025212 and 10872200).

\section{REFERENCES}

1. D. Tabor: Hardness of Metals (Clarendon Press, Oxford, UK, 1951), pp. 73, 76.

2. M. Dao and N. Chollacoop: Computational modeling of the forward and reverse problems in instrumented sharp indentation. Acta Mater. 49, 3899 (2001). 
3. Y.T. Cheng and C.M. Cheng: Can stress-strain relationships be obtained from indentation curves using conical and pyramidal indenters? J. Mater. Res. 14(9), 3493 (1999).

4. X. Chen, N. Ogasawara, M.H. Zhao, and N. Chiba: On the uniqueness of measuring elastoplastic properties from indentation: The indistinguishable mystical materials. J. Mech. Phys. Solids 55(8), 1618 (2007).

5. Y.P. Cao and J. Lu: An energy-based method to extract plastic properties of metal materials from conical indentation tests. J. Mater. Res. 20(5), 1194 (2005).

6. J.H. Ahn and D. Kwon: Derivation of plastic stress-strain relationship from ball indentation: Examination of strain definition and pileup effect. J. Mater. Res. 16, 3170 (2001).

7. N. Choppacoop, M. Dao, and S. Suresh: Depth-sensing instrumented indentation with dual sharp indenters. Acta Mater. 51, 3713 (2003).

8. J.Y. Kim and K.W. Lee: Determination of tensile properties by instrumented indentation technique: Representative stress and strain approach. Surf. Coat. Tech. 201, 4278 (2006).

9. S. Jayaraman, G.T. Hahn, W.C. Oliver, C.A. Rubin, and P.C. Bastias: Determination of monotonic stress strain curve of hard materials from ultra-low-load indentation tests. Int. J. Solids Struct. 35, 365 (1998).

10. B. Taljat, T. Zacharia, and F. Kosel: New analytical procedure to determine stress-strain curve from spherical indentation data. Int. J. Solids Struct. 35(33), 4411 (1998).

11. J.S. Field and M.V. Swain: Determining the mechanical properties of small volumes of material from submicron spherical indentations. J. Mater. Res. 10(1), 101 (1995).
12. Y.P. Cao and J. Lu: A new method to extract the plastic properties of metal materials from an instrumented spherical indentation loading curve. Acta Mater. 52, 4023 (2004).

13. M.H. Zhao, N. Ogasawara, N. Chiba, and X. Chen: A new approach to measure the elastic-plastic properties of bulk materials using spherical indentation. Acta Mater. 54, 23 (2006).

14. P. Jiang, T.H. Zhang, Y.H. Feng, and N.G. Liang: Determination of plastic properties by instrumented spherical indentation: Expanding cavity model and similarity solution approach. J. Mater. Res. 24(3), 1045 (2009).

15. T.H. Zhang, P. Jiang, Y.H. Feng, and R. Yang: Numerical verification for instrumented spherical indentation techniques in determining the plastic properties of materials. J. Mater. Res. 24(12), 3653 (2009).

16. W.C. Oliver and G.M. Pharr: An improved technique for determining hardness and elastic modulus using load and displacement sensing indentation experiments. J. Mater. Res. 7(6), 1546 (1992).

17. L. Liu, N. Ogasawara, N. Chiba, and X. Chen: Can indentation technique measure unique elastoplastic properties? J. Mater. Res. 24(3), 784 (2009).

18. R. Hill, B. Storakers, and A.B. Zdunek: A theoretical study of the Brinell hardness test. Proc. R. Soc. London, Ser. A 423, 301 (1989).

19. ISO 4288:1996. Geometrical product specifications (GPS)Surface texture: Profile method-Rules and procedures for the assessment of surface texture.

20. ISO 14577:2002. Metallic materials-Instrumented indentation test for hardness and materials parameters. 